\title{
Calculation of enhanced scattering peaks in disordered thin slabs with internal reflections
}

\author{
Qianjin Chu, Xiangdong Zhang, and Zhao-Qing Zhang \\ Department of Physics, Hong Kong University of Science and Technology, Clear Water Bay, Kowloon, Hong Kong
}

(Received 14 April 2004; revised manuscript received 11 June 2004; published 23 September 2004)

\begin{abstract}
By performing first-principles calculations, we study theoretically the angular distributions of the intensities of the scattered waves which are reflected from and transmitted through disordered thin slabs with internal reflections in the case when $L<l$ and $\lambda<l$, where $L$ is the thickness of the slab, $l$ is the mean free path, and $\lambda$ is the wavelength inside the slab. We find that the enhanced scattering peak on the transmission side has almost the same structure as the coherent backscattering (CBS) peak on the reflection side when the phase factor $\Phi \equiv k L\left[\sqrt{\left(m^{2}-\sin ^{2} \theta_{i}\right)} / m\right]<1$, where $k=2 \pi / \lambda, m$ is the relative refractive index of the slab, and $\theta_{i}$ is the incident angle. When $\Phi>1$, the enhanced scattering peak on the transmission side shows an oscillatory behavior. The amplitudes of the oscillations increase with $m$ and $\theta_{i}$. It is also found that the widths of the enhanced scattering peaks increase with $L$. This is opposite to the well-known trend in thick slabs, where the width of the CBS peak decreases with $L$. When $L>\lambda$, we find satellite peaks, which are much weaker in intensity than the main enhanced scattering peaks.
\end{abstract}

DOI: 10.1103/PhysRevB.70.125110 PACS number(s): 78.20.-e, 42.25.Bs, 42.25.Dd, 42.25.Kb

\section{INTRODUCTION}

The study of scattering and propagation of waves in random media is an old topic which appeared over one century ago. ${ }^{1,2}$ In it the coherent backscattering (CBS) has attracted a great deal of attention. ${ }^{3-10}$ From the interference between scattered waves, which travel through the disordered sample along different paths, we have learned the fascinating aspects of the multiply scattered wavefield. When waves are scattered from disordered media, within a certain solid angle around the direction of pure backscattering there will be an enhanced intensity or, in other words, the interference of the waves which travel along the same wave path in opposite directions increases the returning probability. This phenomenon is known as weak localization. The properties of the CBS, especially the feature of its cone, have been studied in detail by a large number of experiments and theories., ${ }^{3,4}$ The effects due to internal reflection on the CBS cone have also been investigated by many researchers. ${ }^{5-8}$ The sharpening of the CBS cone has been found when amplification is introduced into the random media. ${ }^{9}$ However in these studies, only thick samples are studied, where the sample thickness $L$ is assumed to be either much larger than the mean free path $l$ or semi-infinite and the wavelength inside the slab $\lambda$ is assumed to be much smaller than $l$ so that the diffusion approximation is valid.

For thin samples $(L<l)$, Fabry-Perot (FP) resonances may also exist. Berkovits and Feng ${ }^{10}$ predicted that the peaks of the enhanced scattering will appear not only on the reflection side in the backscattering direction, but also on the transmission side in the mirror direction of the incident wave. Based on a phenomenological theory, they calculated the angular distribution of the transmitted intensity and found an enhancement factor of 2 in the mirror direction of the incident wave. Furthermore, when $L>\lambda$, it has been predicted that the enhanced scattering may occur not only near the backscattering and mirror directions, but also in other satellite directions both on the reflection and transmission sides. ${ }^{12,13}$ This is because that each wave path inside the slab may be multifold degenerated, and hence the phase difference between the time-reversed paths, i.e., a multiply scattered wave and its time-reversed counter part, may exist on both sides of the slab. However, the calculated satellite peaks in Refs. 12 and 13 were also based on a phenomenological theory, in which the randomness considered was two dimensional instead of three dimensional.

Despite all the interesting phenomena reported previously in disordered thin slabs, a first principles theory is required to give a systematic and quantitative understanding of all these phenomena under a unified picture. For instance, not much is known about the properties of the enhanced scattering peak on the transmission side. It is well known that the enhancement factor is always 2 in the backscattering direction when the single-scattering contribution is not included. 3,4 However, this is not true in general on the transmission side. The interference property of the multip scattered waves on the transmission side is different from that on the reflection side. The time-reversed paths inside the slab are not strictly constructive interference paths on the transmission side in the mirror direction of the incident wave. This can be seen from Fig. 1(a), where a phase difference $2 k\left(z_{2}-z_{1}\right)$ between the time-reversed paths on the transmission side is shown for normally incident waves. Here $k=2 \pi / \lambda$ is the wave number inside the slab and $z_{1}, z_{2}$ are, respectively, the positions of the first and the last scatterers along the $z$ axis. In the backscattering direction, there is no phase difference between the time-reversed paths. In order to have a quantitative understanding of how this phase difference affects the enhanced scattering peak on the transmission side, a first principles study is necessary.

In this work, by using the Bethe-Salpeter equation with internal reflections incorporated, we study theoretically the angular distributions of the intensities of the scattered waves both on the reflection and transmission sides of a thin slab with $L<l$. In calculating the intensities of the scattered waves, we assume weak scattering, i.e., $\lambda<l$, and only con- 


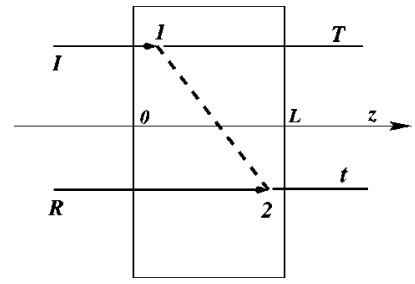

(a) (b)

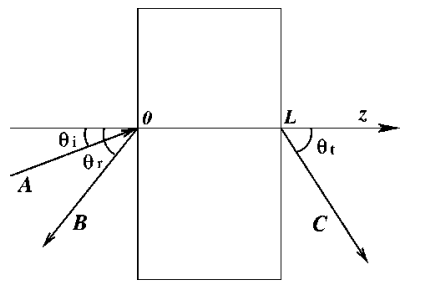

FIG. 1. (a) One important difference between the transmission and reflection sides is demonstrated by several wave paths for normal incidence. On the reflection side, the paths of $I \rightarrow 1 \rightarrow 2 \rightarrow R$ and $R \rightarrow 2 \rightarrow 1 \rightarrow I$ have the same length. But on the transmission side, the paths of $R \rightarrow 2 \rightarrow 1 \rightarrow T$ and $I \rightarrow 1 \rightarrow 2 \rightarrow t$ have different lengths. The difference is $2\left(z_{2}-z_{1}\right)$. (b) $A$ an incident plane wave at incident angle $\theta_{i}, B$ the scattered wave on the reflection side in the direction $\theta_{r}, C$ the scattered wave on the transmission side in the direction $\theta_{t}$.

sider the contributions from the single-scattering diagram, the ladder diagrams (LD), and the maximally crossed diagrams (MCD's). Our results show that when the phase factor $\Phi \equiv k L\left[\sqrt{\left(m^{2}-\sin ^{2} \theta_{i}\right)} / m\right]<1$, where $m$ is the relative refractive index of the slab and $\theta_{i}$ is the incident angle, the transmitted peak has almost the same structure as that of the reflected one. In this case, the enhancement factor on the transmission side $E_{t}$ is close to 2 . When $\Phi>1, E_{t}$ exhibits an oscillatory behavior and approaches one when $\Phi \approx 2.3$ $+n \pi(n=0,1,2, \ldots)$. The amplitudes of the oscillations increase with $m$ and $\theta_{i}$. It is also found that the widths of the enhanced scattering peaks increase with $L$. This is opposite to the well-known trend in thick slabs where the width of the CBS cone decreases with $L$ due to the presence of longer paths. For the case of $L>\lambda$, we find satellite peaks. The satellite peak on the transmission side is smaller than that on the reflection side. They both are much weaker in intensity than the main enhanced scattering peaks. In Sec. II we give the analytical formulas for calculating the angular distributions of the intensities of the scattered waves. The numerical results and discussions will be presented in Sec. III. Section IV gives a brief conclusion of this work.

\section{THEORY}

We consider a plane wave A that is incident on the front surface $z=0$ of a disordered slab in the direction $\left(\theta_{i}, \varphi_{i}\right)$ shown in Fig. 1(b). $B$ and $C$ are, respectively, the scattered waves on the reflection side in the direction $\left(\theta_{r}, \varphi_{r}\right)$ and on the transmission side in the direction $\left(\theta_{t}, \varphi_{t}\right)$. In this work we only consider the azimuthal angles $\varphi_{r}=\varphi_{t}=\pi+\varphi_{i}$. The thickness $L$ of the sample is assumed to be smaller than the mean free path $l$. The refractive index $n$ inside the slab $(0<z<L)$ is larger than or equal to that outside, i.e., $n \equiv m n_{0}$, where $m \geqslant 1$ is the relative refraction index of the slab.

After configurational average, the coherent wave $\left\langle\psi_{i}(\vec{r}, \Omega)\right\rangle$ satisfies the following equations: ${ }^{8,11}$

$$
\begin{gathered}
{\left[\nabla_{\vec{r}}^{2}+\left(k+\frac{i}{2 l}\right)^{2}\right]\left\langle\psi_{i}(\vec{r}, \Omega)\right\rangle=0, \quad 0<z<L,} \\
\left(\nabla_{\vec{r}}^{2}+k_{0}^{2}\right)\left\langle\psi_{i}(\vec{r}, \Omega)\right\rangle=0, \quad z<0 \text { or } z>L,
\end{gathered}
$$

where $k_{0}=\Omega / v_{0}=2 \pi / \lambda_{0}, k=\Omega / v=2 \pi / \lambda$, and $\Omega$ is the wave frequency. $v_{0}=c / n_{0}$ and $v=c / n$ are the wave speeds in different regions, and $\lambda_{0}$ and $\lambda$ are the wavelengths in different regions.

For a plane wave incidence with an amplitude equal to one, the solution of Eq. (1) can be written as ${ }^{8,11}\left\langle\psi_{i}(\vec{r}, \Omega)\right\rangle$ $\equiv\left\langle\phi_{i}(z, \Omega)\right\rangle e^{i \vec{q}_{i} \cdot \vec{\rho}}$ with

$$
\left\langle\phi_{i}(z, \Omega)\right\rangle= \begin{cases}e^{i p_{0 i} z}+\left(\widetilde{p}_{i}-p_{0 i}\right) \phi_{i}^{\text {peak }}\left(\theta_{i}\right)\left(e^{2 i \tilde{p}_{i} L}-1\right) e^{-i p_{0 i} z}, & z<0, \\ 2 p_{0 i} \phi_{i}^{\text {peak }}\left(\theta_{i}\right)\left[e^{i \tilde{p}_{i} z}+r_{i} e^{i \tilde{p}_{i}(2 L-z)}\right], & 0<z<L, \\ \frac{4 p_{0 i} \widetilde{p}_{i}}{\widetilde{p}_{i}+p_{0 i}} \phi_{i}^{\text {peak }}\left(\theta_{i}\right) e^{i \tilde{p}_{i} L+i p_{0 i}(z-L)}, & z>L,\end{cases}
$$

where $\vec{r} \equiv(\vec{\rho}, z), \vec{q}_{i}$ is the incident wave vector in the transverse direction, and $q_{i}=k_{0} \sin \theta_{i}$ in all regions, $p_{0 i}=\sqrt{k_{0}^{2}-q_{i}^{2}}$ $=k_{0} \cos \theta_{i}, r_{i}=\left(\widetilde{p}_{i}-p_{0 i}\right) /\left(\widetilde{p}_{i}+p_{0 i}\right)$, and

$$
\begin{gathered}
\widetilde{p}_{i}=\sqrt{[k+i /(2 l)]^{2}-k_{0}^{2} \sin ^{2} \theta_{i}}, \text { with } \operatorname{Im}\left(\widetilde{p}_{i}\right) \geqslant 0, \\
\phi_{i}^{\text {peak }}\left(\theta_{i}\right)=\frac{1}{\left(\widetilde{p}_{i}+p_{0 i}\right)\left(1-r_{i}^{2} e^{2 i \tilde{p}_{i} L}\right)} .
\end{gathered}
$$

It should be pointed out that the function $\phi_{i}^{\text {peak }}\left(\theta_{i}\right)$ contains the FP interference.
The total intensity of the waves $\left\langle\psi(\vec{r}, \Omega) \psi^{*}(\vec{r}, \Omega)\right\rangle$ can be obtained from the field-field correlation function $\left\langle\psi(\vec{r}, \Omega) \psi^{*}\left(\vec{r}^{\prime}, \Omega\right)\right\rangle$, which satisfies the following BetheSalpeter equation: ${ }^{3,11}$

$$
\begin{aligned}
\left\langle\psi(\vec{r}, \Omega) \psi^{*}\left(\vec{r}^{\prime}, \Omega\right)\right\rangle= & \left\langle\psi_{i}(\vec{r}, \Omega)\right\rangle\left\langle\psi_{i}^{*}\left(\vec{r}^{\prime}, \Omega\right)\right\rangle+\int d \vec{r}_{1} d \vec{r}_{2} d \vec{r}_{3} d \vec{r}_{4} \\
& \times\left\langle G_{\Omega}\left(\vec{r}, \vec{r}_{1}\right)\right\rangle\left\langle G_{\Omega}^{*}\left(\vec{r}^{\prime}, \vec{r}_{2}\right)\right\rangle U\left(\vec{r}_{1}, \vec{r}_{3} ; \vec{r}_{2}, \vec{r}_{4}\right) \\
& \times\left\langle\psi\left(\vec{r}_{3}, \Omega\right) \psi^{*}\left(\vec{r}_{4}, \Omega\right)\right\rangle,
\end{aligned}
$$

where $\left\langle G_{\Omega}\left(\vec{r}, \vec{r}_{1}\right)\right\rangle$ is the averaged single-particle Green's 
function and $U$ stands for the summation of all irreducible vertex functions. Because $\left\langle G_{\Omega}\left(\vec{r}_{1}, \vec{r}_{2}\right)\right\rangle$ possesses the translational invariance in the transverse plane, it can be written as

$$
\left\langle G_{\Omega}\left(\vec{r}_{1}, \vec{r}_{2}\right)\right\rangle=\frac{1}{4 \pi^{2}} \int d \vec{q}\left\langle G\left(z_{1}, z_{2} ; q\right)\right\rangle e^{i \vec{q} \cdot\left(\vec{\rho}_{1}-\vec{\rho}_{2}\right)},
$$

where $\left\langle G\left(z_{1}, z_{2} ; q\right)\right\rangle$ satisfies the following one-dimensional wave equations: ${ }^{8,11}$

$$
\begin{gathered}
{\left[d^{2} / d z_{1}^{2}-q^{2}+\left(k+\frac{i}{2 l}\right)^{2}\right]\left\langle G\left(z_{1}, z_{2} ; q\right)\right\rangle} \\
=\delta\left(z_{1}-z_{2}\right), \quad 0<z_{1}, z_{2}<L, \\
\left(d^{2} / d z_{1}^{2}-q^{2}+k_{0}^{2}\right)\left\langle G\left(z_{1}, z_{2} ; q\right)\right\rangle \\
=0, \quad z_{1}<0 \text { or } z_{1}>L, 0<z_{2}<L .
\end{gathered}
$$

From the above equations, when $0<z_{1}, z_{2}<L$, we obtain

$$
\begin{aligned}
\left\langle G\left(z_{1}, z_{2} ; q\right)\right\rangle= & -i \phi^{\text {peak }}(q)\left[e^{i \widetilde{p}_{q}\left|z_{1}-z_{2}\right|}+r_{q} e^{i \widetilde{p}_{q}\left(z_{1}+z_{2}\right)}\right. \\
& \left.+r_{q} e^{i \tilde{p}_{q}\left[2 L-\left(z_{1}+z_{2}\right)\right]}+r_{q}^{2} e^{i \widetilde{p}_{q}\left[2 L-\left|z_{1}-z_{2}\right|\right]}\right],
\end{aligned}
$$

where $\widetilde{p}_{q}=\sqrt{(k+i /(2 l))^{2}-q^{2}}$ with $\operatorname{Im}\left(\widetilde{p}_{q}\right) \geqslant 0, r_{q}=\left(\widetilde{p}_{q}-\widetilde{p}_{0 q}\right) /$ $\left(\widetilde{p}_{q}+\widetilde{p}_{0 q}\right)$, and $\widetilde{p}_{0 q}=\sqrt{k_{0}^{2}-q^{2}}$ with $\operatorname{Im}\left(\widetilde{p}_{0 q}\right) \geqslant 0$. Similar to $\phi_{i}^{\text {peak }}\left(\theta_{i}\right), \phi^{\text {peak }}(q)=1 / 2 \widetilde{p}_{q}\left(1-r_{q}^{2} e^{2 i \widetilde{p}_{q} L}\right)$ also contains the FP interference. For $z_{1}$ outside the disordered slab, we consider only the propagating waves. In this case, $q=q_{o}=k_{0} \sin \theta_{o}$, and the subscript $o=r$ or $t$ stands for that $z_{1}$ on the reflection or transmission side. The solution of Eq. (6) becomes

$$
\begin{aligned}
\left\langle G\left(z_{1}, z_{2} ; q_{r}\right)\right\rangle= & -i \phi_{r}^{\text {peak }}\left(\theta_{r}\right)\left[e^{i \widetilde{p}_{r} z_{2}}+r_{r} e^{i \widetilde{p}_{r}\left(2 L-z_{2}\right)}\right] \\
& \times e^{-i p_{0 r} z_{1}}, \quad z_{1}<0,0<z_{2}<L, \\
\left\langle G\left(z_{1}, z_{2} ; q_{t}\right)\right\rangle= & -i \phi_{t}^{\text {peak }}\left(\theta_{t}\right)\left[e^{i \widetilde{p}_{t}\left(L-z_{2}\right)}+r_{t} e^{i \widetilde{p}_{t}\left(L+z_{2}\right)}\right] \\
& \times e^{i p_{0 t}\left(z_{1}-L\right)}, \quad z_{1}>L, 0<z_{2}<L,
\end{aligned}
$$

where $\quad p_{0 o}=k_{0} \cos \theta_{o}, \widetilde{p}_{o}=\sqrt{[k+i /(2 l)]^{2}-k_{0}^{2} \sin ^{2} \theta_{o}} \quad$ with $\operatorname{Im}\left(\widetilde{p}_{o}\right) \geqslant 0, r_{o}=\left(\widetilde{p}_{o}-p_{0 o}\right) /\left(\widetilde{p}_{o}+p_{0 o}\right)$, and $\phi_{o}^{\text {peak }}\left(\theta_{o}\right)=1 /\left(\widetilde{p}_{o}\right.$ $\left.+p_{0 o}\right)\left(1-r_{o}^{2} e^{2 i \tilde{p}_{0} L}\right)$.

In the casse of weak scattering considered here, i.e., $k l>2 \pi$, or $\lambda<l$, we can approximate Eq. (4) as ${ }^{3}$

$$
\begin{aligned}
& \left\langle\psi(\vec{r}, \Omega) \psi^{*}(\vec{r}, \Omega)\right\rangle \\
& =\left\langle\psi_{i}(\vec{r}, \Omega)\right\rangle\left\langle\psi_{i}^{*}(\vec{r}, \Omega)\right\rangle+\int d \vec{r}_{1}\left\langle G_{\Omega}\left(\vec{r}, \vec{r}_{1}\right)\right\rangle \\
& \times\left\langle G_{\Omega}^{*}\left(\vec{r}, \vec{r}_{1}\right)\right\rangle \frac{4 \pi}{l}\left\langle\psi_{i}\left(\vec{r}_{1}, \Omega\right)\right\rangle\left\langle\psi_{i}^{*}\left(\vec{r}_{1}, \Omega\right)\right\rangle \\
& \quad+\int d \vec{r}_{1} d \vec{r}_{2}\left\langle G_{\Omega}\left(\vec{r}, \vec{r}_{1}\right)\right\rangle\left\langle G_{\Omega}^{*}\left(\vec{r}, \vec{r}_{1}\right)\right\rangle F\left(\vec{r}_{1}, \vec{r}_{2}\right) \\
& \times\left\langle\psi_{i}\left(\vec{r}_{2}, \Omega\right)\right\rangle\left\langle\psi_{i}^{*}\left(\vec{r}_{2}, \Omega\right)\right\rangle+\int d \vec{r}_{1} d \vec{r}_{2}\left\langle G_{\Omega}\left(\vec{r}, \vec{r}_{1}\right)\right\rangle \\
& \quad \times\left\langle G_{\Omega}^{*}\left(\vec{r}, \vec{r}_{2}\right)\right\rangle F\left(\vec{r}_{1}, \vec{r}_{2}\right)\left\langle\psi_{i}\left(\vec{r}_{2}, \Omega\right)\right\rangle\left\langle\psi_{i}^{*}\left(\vec{r}_{1}, \Omega\right)\right\rangle,
\end{aligned}
$$

where the scattering is assumed to be pointlike. The second term of the right-hand side of Eq. (10) represents the singly scattered intensity, the third term stands for the multiply scattered intensities, which come from the LD, and the fourth term stands for the enhanced intensities, which come from the MCD. The function $F\left(\vec{r}_{1}, \vec{r}_{2}\right)$ in Eq. (10) represents the $\mathrm{LD}$ and satisfies the following integral equation:

$$
F\left(\vec{r}_{1}, \vec{r}_{2}\right)=\frac{16 \pi^{2}}{l^{2}} A\left(\vec{r}_{1}, \vec{r}_{2}\right)+\frac{4 \pi}{l} \int d \vec{r}_{3} A\left(\vec{r}_{1}, \vec{r}_{3}\right) F\left(\vec{r}_{3}, \vec{r}_{2}\right)
$$

with the kernel

$$
A\left(\vec{r}_{1}, \vec{r}_{2}\right)=\left\langle G_{\Omega}\left(\vec{r}_{1}, \vec{r}_{2}\right)\right\rangle\left\langle G_{\Omega}^{*}\left(\vec{r}_{1}, \vec{r}_{2}\right)\right\rangle .
$$

Since the functions $A$ and $F$ are also translational invariant in the transverse plane, i.e., the same as $\left\langle G_{\Omega}\left(\vec{r}_{1}, \vec{r}_{2}\right)\right\rangle$, we can use the following relations:

$$
\begin{aligned}
& A\left(\vec{r}_{1}, \vec{r}_{2}\right)=\frac{1}{4 \pi^{2}} \int d \vec{q} A\left(z_{1}, z_{2} ; q\right) e^{i \vec{q} \cdot\left(\vec{\rho}_{1}-\vec{\rho}_{2}\right)}, \\
& F\left(\vec{r}_{1}, \vec{r}_{2}\right)=\frac{1}{4 \pi^{2}} \int d \vec{q} F\left(z_{1}, z_{2} ; q\right) e^{i \vec{q} \cdot\left(\vec{\rho}_{1}-\vec{\rho}_{2}\right)},
\end{aligned}
$$

to rewrite Eqs. (11) and (12) as

$$
\begin{aligned}
A\left(z_{1}, z_{2} ; q\right)=\frac{1}{4 \pi^{2}} \int d \vec{q}_{1}\left\langle G\left(z_{1}, z_{2} ; q_{1}\right)\right\rangle\left\langle G^{*}\left(z_{1}, z_{2} ;\left|\vec{q}-\vec{q}_{1}\right|\right)\right\rangle, & \\
F\left(z_{1}, z_{2} ; q\right)= & \frac{16 \pi^{2}}{l^{2}} A\left(z_{1}, z_{2} ; q\right) \\
& +\frac{4 \pi}{l} \int d z_{3} A\left(z_{1}, z_{3} ; q\right) F\left(z_{3}, z_{2} ; q\right) .
\end{aligned}
$$

Thus, the total intensity of the scattered waves given in Eq. (10) can be reexpressed as

$$
\begin{aligned}
I(\vec{r})= & \left\langle\psi(\vec{r}, \Omega) \psi^{*}(\vec{r}, \Omega)\right\rangle-\left\langle\psi_{i}(\vec{r}, \Omega)\right\rangle\left\langle\psi_{i}^{*}(\vec{r}, \Omega)\right\rangle \\
= & \frac{1}{\pi l} \int d z_{1} d \vec{q}_{o}\left\langle G\left(z, z_{1} ; q_{o}\right)\right\rangle\left\langle G^{*}\left(z, z_{1} ; q_{o}\right)\right\rangle\left\langle\phi_{i}\left(z_{1}, \Omega\right)\right\rangle \\
& \times\left\langle\phi_{i}^{*}\left(z_{1}, \Omega\right)\right\rangle+\frac{1}{4 \pi^{2}} \int d z_{1} d z_{2} d \vec{q}_{o}\left\langle G\left(z, z_{1} ; q_{o}\right)\right\rangle \\
& \times\left\langle G^{*}\left(z, z_{1} ; q_{o}\right)\right\rangle F\left(z_{1}, z_{2} ; 0\right)\left\langle\phi_{i}\left(z_{2}, \Omega\right)\right\rangle\left\langle\phi_{i}^{*}\left(z_{2}, \Omega\right)\right\rangle \\
& +\frac{1}{4 \pi^{2}} \int d z_{1} d z_{2} d \vec{q}_{o}\left\langle G\left(z, z_{1} ; q_{o}\right)\right\rangle \\
& \times\left\langle G^{*}\left(z, z_{2} ; q_{o}\right)\right\rangle F\left(z_{1}, z_{2} ;\left|\vec{q}_{i}+\vec{q}_{o}\right|\right)\left\langle\phi_{i}\left(z_{2}, \Omega\right)\right\rangle\left\langle\phi_{i}^{*}\left(z_{1}, \Omega\right)\right\rangle,
\end{aligned}
$$

where $\vec{q}_{o}$ is the vector of the outgoing wave in the transverse direction. When $|z| \rightarrow \infty$, all the evanescent waves do not contribute and $I(\vec{r})$ in Eq. (15) becomes $\vec{r}$ independent. For propagating waves, we can write $q_{o}$ as $q_{o}=k_{0} \sin \theta_{o}$, where $\theta_{o}$ is the outgoing angle. Using $d \vec{q}_{o}=d q_{o} \cdot q_{o} d \varphi_{o}$ $=k_{0} \cos \theta_{o} d \theta_{o} k_{0} \sin \theta_{o} d \varphi_{o}=k_{0}^{2} \cos \theta_{o} d \Omega_{o}, I(\vec{r})$ can be formally expressed as 


$$
I(\vec{r})=\int d \Omega_{o} I\left(\theta_{i}, \varphi_{i} ; \theta_{o}, \varphi_{o}\right),
$$

where $\varphi_{i}$ and $\varphi_{o}$ are azimuthal angles of the incoming and outgoing waves, respectively. $I\left(\theta_{i}, \varphi_{i} ; \theta_{o}, \varphi_{o}\right)$ can be considered as the intensity of the scattered waves in the direction $\left(\theta_{o}, \varphi_{o}\right)$ when the observation position is far from the slab and is generally dependent on $\theta_{i}, \varphi_{i}$ and $\theta_{o}, \varphi_{o}$. In this work, we only study the case of $\varphi_{o}-\varphi_{i}=\pi$, meanwhile, $I\left(\theta_{i}, \varphi_{i} ; \theta_{o}, \pi+\varphi_{i}\right)$ is independent of $\varphi_{i}$.

If we denote the intensity of the scattered waves on the reflection side as $I_{o}\left(\theta_{i}, \theta_{o}\right)=I_{r}\left(\theta_{i}, \theta_{r}\right) \equiv I\left(\theta_{i}, \varphi_{i} ; \pi-\theta_{r}, \pi+\varphi_{i}\right)$ and on the transmission side as $I_{o}\left(\theta_{i}, \theta_{o}\right)=I_{t}\left(\theta_{i}, \theta_{t}\right)$ $\equiv I\left(\theta_{i}, \varphi_{i} ; \theta_{t}, \pi+\varphi_{i}\right)$, after substituting Eqs. (8) and (9) into Eq. (15) and using Eq. (16), we find

$$
\begin{aligned}
I_{o}\left(\theta_{i}, \theta_{o}\right)= & \frac{\cos \theta_{o}}{4 \pi l} \int d z_{1} \phi_{o}\left(z_{1}\right) \phi_{o}^{*}\left(z_{1}\right)\left\langle\phi_{i}\left(z_{1}, \Omega\right)\right\rangle\left\langle\phi_{i}^{*}\left(z_{1}, \Omega\right)\right\rangle \\
& +\frac{\cos \theta_{o}}{(4 \pi)^{2}} \int d z_{1} d z_{2} \phi_{o}\left(z_{1}\right) \phi_{o}^{*}\left(z_{1}\right) F\left(z_{1}, z_{2} ; 0\right) \\
& \times\left\langle\phi_{i}\left(z_{2}, \Omega\right)\right\rangle\left\langle\phi_{i}^{*}\left(z_{2}, \Omega\right)\right\rangle \\
& +\frac{\cos \theta_{o}}{(4 \pi)^{2}} \int d z_{1} d z_{2} \phi_{o}\left(z_{1}\right) \phi_{o}^{*}\left(z_{2}\right) F\left(z_{1}, z_{2} ; k_{0} \mid \sin \theta_{o}\right. \\
& \left.-\sin \theta_{i} \mid\right)\left\langle\phi_{i}\left(z_{2}, \Omega\right)\right\rangle\left\langle\phi_{i}^{*}\left(z_{1}, \Omega\right)\right\rangle,
\end{aligned}
$$

where the subscript $o=r$ or $t$ stands for the scattered waves on the reflection or transmission side and

$$
\begin{gathered}
\phi_{r}\left(z_{1}\right)=2 k_{0} \phi_{r}^{\text {peak }}\left(\theta_{r}\right)\left[e^{i \widetilde{p}_{r} z_{1}}+r_{r} e^{i \widetilde{p}_{r}\left(2 L-z_{1}\right)}\right], \\
\phi_{t}\left(z_{1}\right)=2 k_{0} \phi_{t}^{\text {peak }}\left(\theta_{t}\right)\left[e^{i \widetilde{p}_{t}\left(L-z_{1}\right)}+r_{t} e^{i \widetilde{p}_{t}\left(L+z_{1}\right)}\right] .
\end{gathered}
$$

In all the discussions later, we use $I_{r}^{s}\left(\theta_{i}, \theta_{r}\right)$ or $I_{t}^{s}\left(\theta_{i}, \theta_{t}\right)$ to denote the first term of $I_{o}\left(\theta_{i}, \theta_{o}\right)$. The second and third terms of $I_{o}\left(\theta_{i}, \theta_{o}\right)$ are denoted by $I_{r}^{l}\left(\theta_{i}, \theta_{r}\right)$ and $I_{r}^{c}\left(\theta_{i}, \theta_{r}\right)$, respectively, on the reflection side and $I_{t}^{l}\left(\theta_{i}, \theta_{t}\right)$ and $I_{t}^{c}\left(\theta_{i}, \theta_{t}\right)$, respectively, on the transmission side.

\section{NUMERICAL RESULTS AND DISCUSSION}

The function $l^{2} F\left(z_{1}, z_{2} ; q\right)$ is dimensionless. It can be obtained by solving Eqs. (14) numerically for $0<z_{1}, z_{2}<L$ at given $q, n_{0}, m, k_{0} l$, and $L / l$, where $k_{0}$ is the wave number outside the slab. In this work, we set $n_{0}=1$. After $F\left(z_{1}, z_{2} ; q\right)$ is obtained, from Eqs. (2), (18), and (17), we can calculate the scattered intensity $I_{o}\left(\theta_{i}, \theta_{o}\right)$ in the outgoing direction $\theta_{o}$ at given $\theta_{i}, m, k_{0} l$, and $L / l$. From Eq. (17), we can see that the property of the scattered waves is determined by the functions $\phi_{o}(z),\left\langle\phi_{i}(z, \Omega)\right\rangle$ and $F\left(z_{1}, z_{2} ; q\right)$. The functions $\phi_{o}(z)$ and $\left\langle\phi_{i}(z, \Omega)\right\rangle$ contain, respectively, the peaked functions $\phi_{o}^{\text {peak }}\left(\theta_{o}\right)$ and $\phi_{i}^{\text {peak }}\left(\theta_{i}\right)$, arising from the FP interference. Thus it is expected that as $\theta_{o}$ changes from 0 to $\pi / 2$, the function $I_{o}\left(\theta_{i}, \theta_{o}\right)$ will show nonmonotonic behavior that comes from the FP resonances when $L \geqslant \lambda / 2$. The third term of $I_{o}\left(\theta_{i}, \theta_{o}\right)$ contains not only the FP interference through $\left\langle\phi_{i}(z, \Omega)\right\rangle$ and $\phi_{o}(z)$, but also the constructive interference of

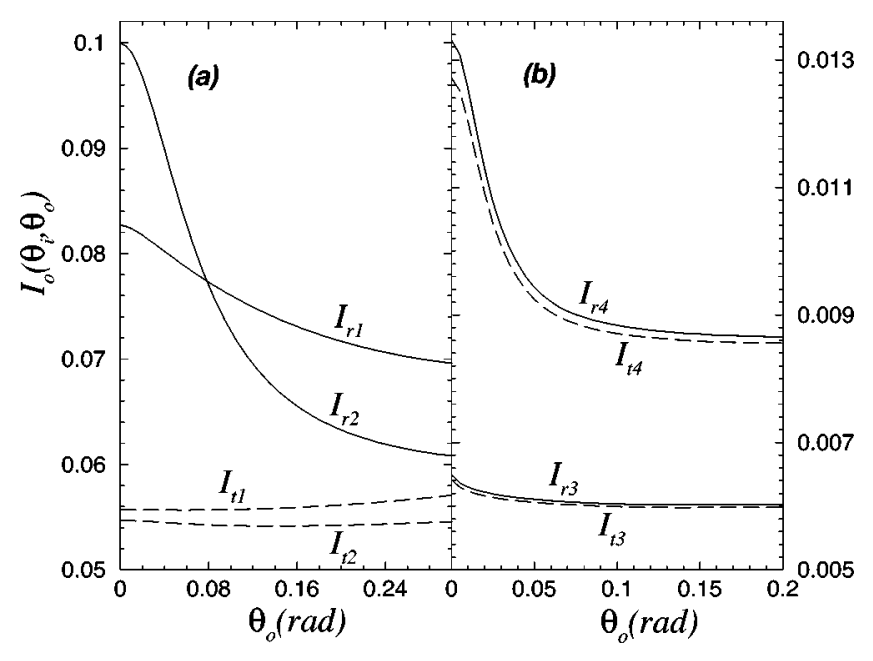

FIG. 2. Intensity $I_{o}\left(\theta_{i}, \theta_{o}\right)$ of the scattered waves (in units of the incident wave intensity), as the function of the outgoing angle $\theta_{o}$ for normal incidence $\left(\theta_{i}=0\right)$. (a) $k_{0} l=8, L / l=0.6, m=1$, and $m=1.6$. $I_{r 1}, I_{t 1}$ denote the angular distributions of the scattered intensities on the reflection and transmission sides, respectively, for the case of $m=1$ and $\left(I_{r 2}, I_{t 2}\right)$ for the case of $m=1.6$. (b) $k_{0} l=8, L / l=0.06, m$ $=1\left(I_{r 3}, I_{t 3}\right)$, and $m=1.6\left(I_{r 4}, I_{t 4}\right)$.

multiply scattered random fields through $F\left(z_{1}, z_{2} ; q\right)$. In the case of $L>\lambda$, the function $\left\langle G\left(z_{1}, z_{2} ; q\right)\right\rangle$ contains at least two quantized eigenmodes due to total internal reflection when $q$ is varied from $k_{0}$ to $k$. The constructive interference between two eigenmodes through the function $F\left(z_{1}, z_{2} ; q\right)$ gives rise to a satellite peak.

\section{A. General properties of the enhanced scattering peaks and effects due to $\mathrm{FP}$ resonances}

For the sake of giving a simple picture of the enhanced scatterings both on the reflection and transmission sides in thin slabs with internal reflection, in Fig. 2 we show the angular distributions of the scattered intensities $I_{r}\left(\theta_{i}, \theta_{r}\right)$ and $I_{t}\left(\theta_{i}, \theta_{t}\right)$ for some typical cases of normal incidence $\left(\theta_{i}=0\right)$, $k_{0} l=8, m=1$ and 1.6 , with (a) $L / l=0.6$ and (b) $L / l=0.06$. Since the amplitude of the incident wave is chosen as unity, all the intensities plotted in the figures shown in this work are in units of the incident wave intensity. From Fig. 2, we can see that the scattered intensities in the case of $L / l$ $=0.06$ are in general smaller than those in the case of $L / l$ $=0.6$. This is due to the fact that the scattered intensity comes from the decay of the coherent wave, and is proportional to $\left(1-e^{-L / l}\right)$. Thus the scattered intensity at $L / l=0.6$ should be greater than that at $L / l=0.06$ by a factor $\left(1-e^{-0.6}\right) /$ $\left(1-e^{-0.06}\right) \approx 7.7$, which is precisely the ratio of the scattered intensities in the backscattering direction in the two cases of $m=1.6$ shown in Fig. 2, i.e., $I_{r 2} / I_{r 4} \cong 7.7$ at $\theta_{o}=0$. In the case of the thinner slab $(L / l=0.06)$, we find that the structures of the enhanced peaks on the reflection and transmission sides are very similar to each other for both $m=1$, where $k L$ $=0.48$, and $m=1.6$, where $k L=0.768$. However, for the thicker slab $(L / l=0.6)$, the peaks of the enhanced scattering on the transmission side cannot be seen in the figure for both 


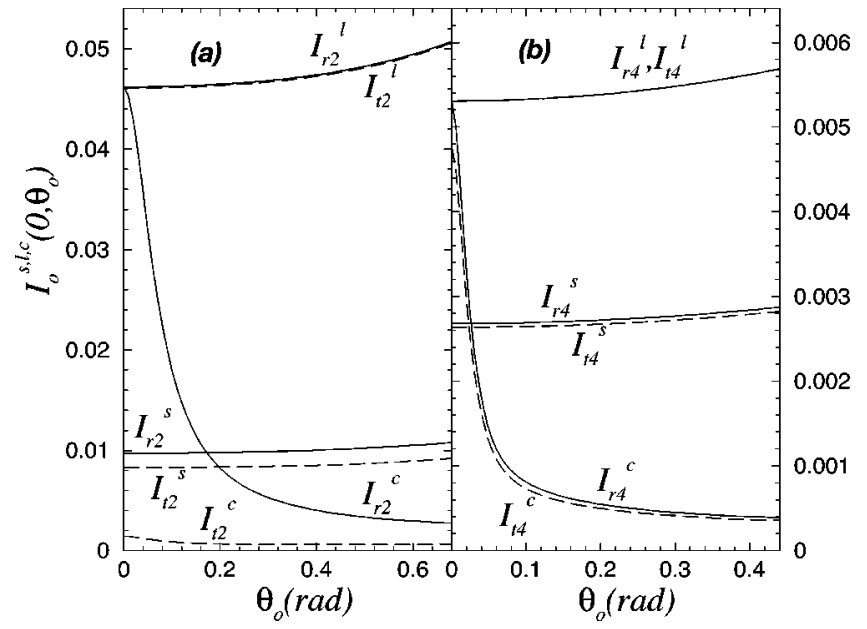

FIG. 3. Intensities $I_{o}^{s, l, c}\left(0, \theta_{o}\right)$ of the scattered waves (in units of the incident wave intensity), as the functions of the outgoing angle $\theta_{o}$, where $\theta_{o}=\theta_{r}$ (or $\theta_{t}$ ) on the reflection (or transmission) side. The values of $k_{0} l, L / l$ in (a) and (b) are same as those in Figs. 2(a) and 2(b), respectively, and $m=1.6 . I_{r}^{s, l, c}=I_{r}^{s, l, c}\left(0, \theta_{r}\right), I_{t}^{s, l, c}=I_{t}^{s, l, c}\left(0, \theta_{t}\right)$.

cases of $m=1(k L=4.8)$ and $m=1.6(k L=7.68)$. This shows clearly that, in the case of normal incidence, it is necessary to have a small value of $k L$ to produce a significant enhanced scattering peak on the transmission side. Furthermore, for the thinner slab $(L / l=0.06)$, although the enhanced peaks on the transmission and reflection sides have almost the same structure, they both are very weak when the system has no internal reflection. This is because the presence of internal reflection tends to trap the waves inside the slab and increases their path lengths and, therefore, produces sharper enhanced scattering peaks. A more systematic discussion on the behavior of the enhanced scattering peak on the transmission side will be given in the next subsection.

In order to give a quantitative discussion of the enhanced scattering peaks, it is necessary to decompose the scattered intensities $I_{o}\left(0, \theta_{o}\right)$ into the singly scattered intensities $I_{o}^{s}\left(0, \theta_{o}\right)$, the multiply scattered intensities $I_{o}^{l}\left(0, \theta_{o}\right)$, and the enhanced intensities $I_{o}^{c}\left(0, \theta_{o}\right)$. For the two cases with $m$ $=1.6$ shown in Figs. 2(a) and 2(b), the decomposed intensities are plotted in Figs. 3(a) and 3(b), respectively. It is clearly seen from Fig. 3 that the functions $I_{r}^{l}\left(0, \theta_{r}\right)$ and $I_{t}^{l}\left(0, \theta_{t}\right)$ are almost identical for thin slabs considered here. They both increase slightly with increasing $\theta_{o}$. This is due to the presence of FP interference in the system. Similar is true for the functions $I_{r}^{s}\left(0, \theta_{r}\right)$ and $I_{t}^{s}\left(0, \theta_{t}\right)$. Since these functions are relatively flat in the cone region of the enhanced scattering peaks, say $I_{r}^{c}\left(0, \theta_{r}\right)$, the effect due to FP interference on the structure of the enhanced scattering peak is insignificant except in the tail region. If we define the ratio of $\left[I_{o}^{c}(0,0)\right.$ $\left.+I_{o}^{l}(0,0)\right] / I_{o}^{l}(0,0)$ as the enhancement factor $E_{o}$ it is clearly seen that $E_{r}$ is always 2, whereas $E_{t}$ is always smaller than 2. The value of $E_{t}$ drops from $1.90(\cong 1+0.00477 / 0.00531)$ for the case of $k L=0.768$ shown in Fig. 3(b) to $1.03(\cong 1$ $+0.00145 / 0.0462)$ for the case of $k L=7.68$ shown in Fig. 3(a).

In order to see the general behavior of $E_{t}$, we first plot in Fig. 4 the intensities $I_{r}^{l}(0,0)$ and $I_{t}^{l, c}(0,0)$ in the backscatter-

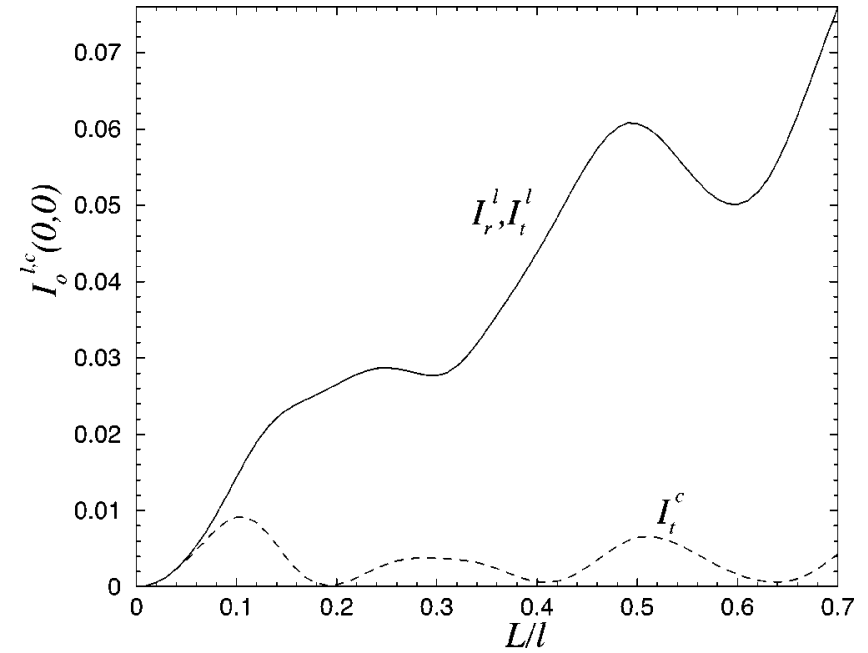

FIG. 4. Intensities $I_{o}^{l, c}(0,0)$ of the scattered waves (in units of the incident wave intensity), as the functions of the thickness $L / l$, with $m=1.6$ and $k_{0} l=8 . I_{r}^{l}=I_{r}^{l}(0,0), I_{t}^{l}=I_{t}^{l}(0,0), I_{t}^{c}=I_{t}^{c}(0,0)$.

ing and mirror directions of the incident wave for normal incidence as functions of the thickness $L / l$, for fixed $m$ $=1.6$ and $k_{0} l=8$. It should be noted that $I_{r}^{c}(0,0)$ is identical to $I_{r}^{l}(0,0)$ and the curves $I_{r}^{l}(0,0)$ and $I_{t}^{l}(0,0)$ are very close and is undiscernible from the figure. It is found that all these decomposed intensities show oscillatory behaviors. However, the positions of peaks and valleys of $I_{r}^{l}$ and $I_{t}^{l}$ are not exactly the same as that of $I_{t}^{c}$. The former arises from FP resonances through the functions $\phi_{o}(z)$ and $\left\langle\phi_{i}(z, \Omega)\right\rangle$ in Eq. (17), whereas the later has additional interference effect arising from the extra phase shown in Fig. 1(a). This additional effect will be discussed in more details later by studying the ratio of the intensities $I_{t}^{c}(0,0)$ and $I_{t}^{l}(0,0)$ so that oscillations due to FP interference can be removed. Apart from these oscillations, both $I_{r}^{l}(0,0)$ and $I_{t}^{l}(0,0)$ increase with $L / l$ due to the increase of source intensity, whereas $I_{t}^{c}(0,0)$ remains roughly unchanged. It is interesting to point out that $I_{t}^{c}(0,0)$ approaches zero at certain values of $L / l$, which are nearly periodic. In Fig. 5, the same intensities $I_{o}^{l, c}(0,0)$ are plotted as functions of $k_{0} l$ for fixed $m=1.6$ and $L / l=0.03$. Similar oscillatory behaviors are found.

\section{B. The behavior of enhancement factor on the transmission side}

The extra phase shown in Fig. 1(a) can generally be understood as follows. The phase difference between the timereversed paths is $\left(\vec{k}_{i}+\vec{k}_{f}\right) \cdot\left(\vec{r}_{2}-\vec{r}_{1}\right)$ for the scattered waves both on the reflection and transmission sides, where $\vec{k}_{i}$ and $\vec{k}_{f}$ are, respectively, the wave vectors inside the slab for incident and outgoing scattered waves and $\vec{r}_{1}, \vec{r}_{2}$ are, respectively, the positions at which the waves are scattered by random medium at the first time and the last time. In the backscattering direction $\vec{k}_{f}=-\vec{k}_{i}$ the phase difference is zero for $I_{r}^{c}\left(\theta_{i}, \theta_{r}\right)$ when $\theta_{r}=\theta_{i}$, leading to an enhancement factor $E_{r}=2$. But in the mirror direction of the incident wave, $\vec{k}_{i}+\vec{k}_{f} \neq 0$ and there is a phase difference $2 k\left[\sqrt{\left(m^{2}-\sin ^{2} \theta_{i}\right)} / m\right]\left(z_{2}-z_{1}\right)$ for 


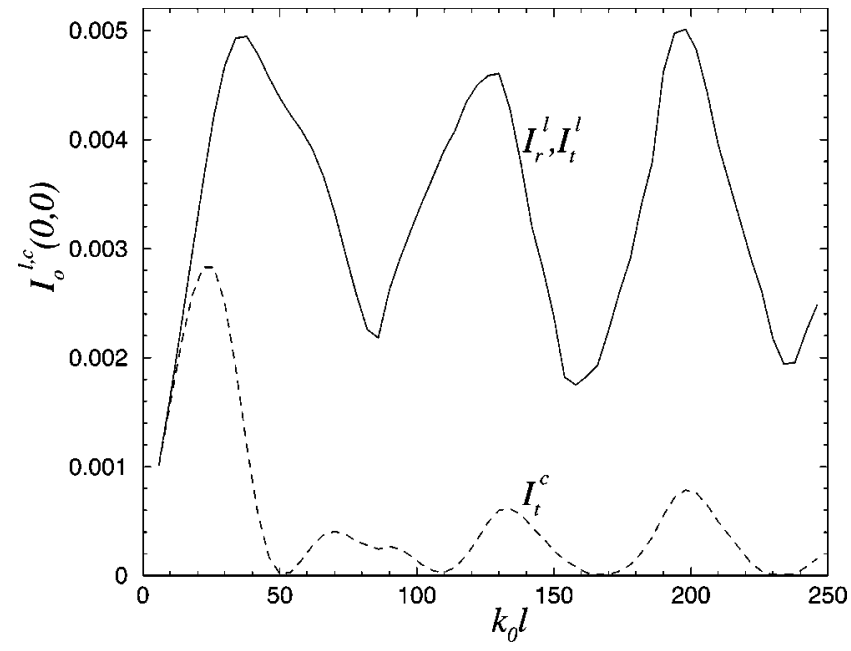

FIG. 5. Intensities $I_{o}^{l, c}(0,0)$ of the scattered waves (in units of the incident wave intensity), as the functions of the dimensionless wave number $k_{0} l$, with $m=1.6$ and $L / l=0.03 . \quad I_{r}^{l}=I_{r}^{l}(0,0), \quad I_{t}^{l}$ $=I_{t}^{l}(0,0), I_{t}^{c}=I_{t}^{c}(0,0)$.

$I_{t}^{c}\left(\theta_{i}, \theta_{t}\right)$ when $\theta_{t}=\theta_{i}$. For normal incidence, the phase difference becomes $2 k\left(z_{2}-z_{1}\right)$ as shown in Fig. 1(a). Hence on the transmission side, the scattered waves which come from the time-reversed paths inside the slab are not strictly constructive interference. This makes $E_{t}<2$.

The phase difference $2 k\left[\sqrt{\left(m^{2}-\sin ^{2} \theta_{i}\right)} / m\right]\left(z_{2}-z_{1}\right)$ in $I_{t}^{c}\left(\theta_{i}, \theta_{i}\right)$ allows us to introduce a convenient phase variable $\Phi \equiv k L\left[\sqrt{\left(m^{2}-\sin ^{2} \theta_{i}\right)} / m\right]$ to describe the behavior of $E_{t}$. In curves 1 and 2 of Fig. 6, we plot the ratios of the intensities $I_{t}^{c}\left(\theta_{i}, \theta_{i}\right)$ and $I_{t}^{l}\left(\theta_{i}, \theta_{i}\right)$ shown in Figs. 4 and 5, respectively, as functions of $\Phi$, i.e., $R \equiv I_{t}^{c}\left(\theta_{i}, \theta_{i}\right) / I_{t}^{l}\left(\theta_{i}, \theta_{i}\right) \equiv E_{t}-1$ with $\theta_{i}$

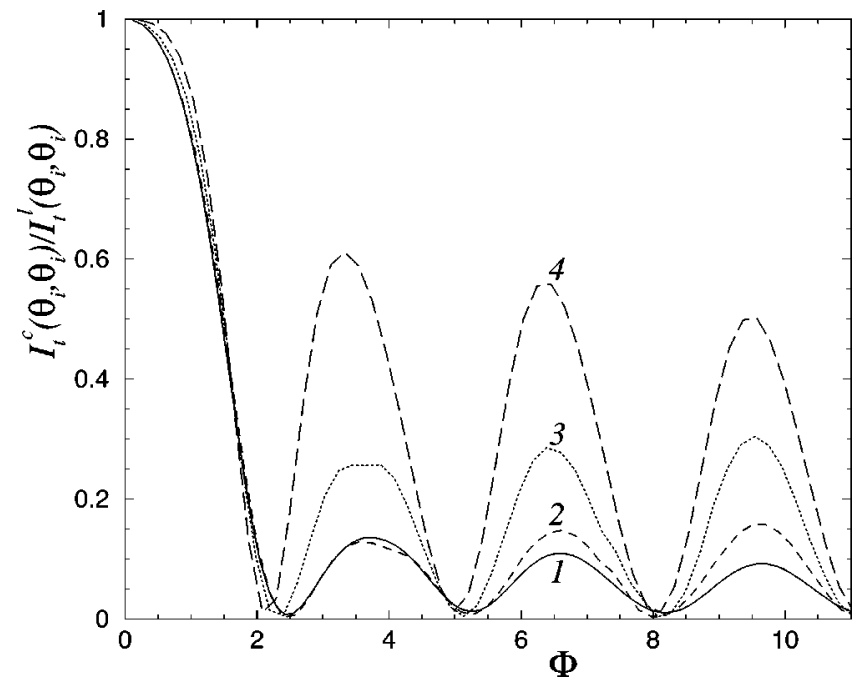

FIG. 6. Intensities's ratio $I_{t}^{c}\left(\theta_{i}, \theta_{i}\right) / I_{t}^{l}\left(\theta_{i}, \theta_{i}\right)$ of the scattered waves, as the function of the phase factor $\Phi$, where the solid line (1) denotes the case of $\theta_{i}=0, m=1.6, k_{0} l=8$, and $L / l$ is assumed to be varied, the dashed line (2) denotes the case of $\theta_{i}=0, m=1.6$, $L / l=0.03$, and $k_{0} l$ is assumed to be varied, the dotted line (3) denotes the case of $\theta_{i}=0, m=2, L / l=0.03$, and $k_{0} l$ is assumed to be varied, and the long dashed line (4) denotes the case of $\theta_{i}$ $=1.2(\mathrm{rad}), m=1.6, k_{0} l=16$, and $L / l$ is assumed to be varied.

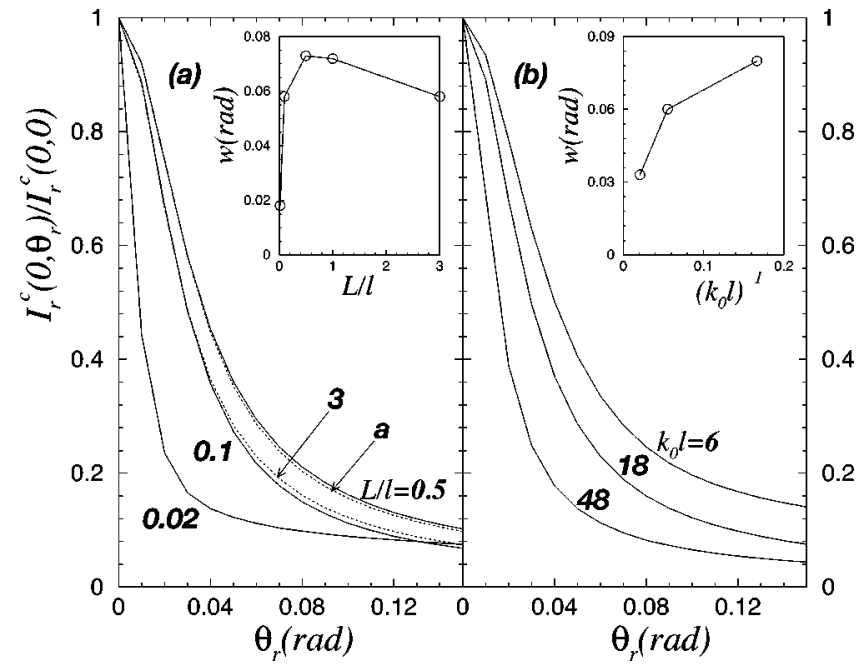

FIG. 7. Normalized intensity $I_{r}^{c}\left(0, \theta_{r}\right) / I_{r}^{c}(0,0)$ of the scattered waves, as the function of the outgoing angle $\theta_{r}$, with $m=1.6$ and (a) $k_{0} l=16, L / l=0.02,0.1,0.5,1,3$, (b) $L / l=0.1, k_{0} l=6,18,48$. Where the numbers in (a) and (b) denote the values of $L / l$ and $k_{0} l$, respectively. The inset in (a) shows the relation between the FWHM $w$ and $L / l$ at given condition in (a). The inset in (b) shows the relation between the FWHM $w$ and $\left(k_{0} l\right)^{-1}$ at given condition in (b).

$=0$. These ratios remove the oscillations due to FP resonances. From these curves, it is easy to see that $R$ is close to 1 when $\Phi<1$. The case shown in Fig. 3(b) falls into this region. When $\Phi>2.3, R$ behaves as a periodical function of $\Phi$ with a period approximately equal to $\pi$. Furthermore, when $\Phi \approx 2.3+n \pi(n=0,1,2, \ldots), R$ is close to zero. The values of $\Phi$ at which $R$ are peaked are $n \pi$ when $\Phi>2.3$, where $n=1,2,3, \ldots$. The heights of these peaks can be roughly estimated by assuming the function $F\left(z_{1}, z_{2} ; 0\right)$ to be a smooth function of $z_{1}$ and $z_{2}$ so that the integrations in Eq. (17) can be performed. As a result, we find, when $\Phi=n \pi(n \geqslant 1)$, $R \approx R_{p} \equiv 4 \gamma^{2} /\left(1+\gamma^{2}\right)^{2}$, where $\gamma=\left(\sqrt{m^{2}-\sin ^{2} \theta_{i}}-\cos \theta_{i}\right) /$ $\left(\sqrt{m^{2}-\sin ^{2} \theta_{i}}+\cos \theta_{i}\right)$. This result suggests that the height of the peaks increases with the internal reflection $m$ and incident angle $\theta_{i}$. In the limits of $m \rightarrow \infty$ or $\theta_{i} \rightarrow \pi / 2$, we have $\gamma \rightarrow 1$ and $R_{p} \rightarrow 1$. To check this, we recalculate the curves 1 and 2 of Fig. 6 using different parameters and plot the results as curves 3 and 4 . In curve 3, we replace the value of $m$ in curve 2 from 1.6 to 2 , whereas in curve 4 , the incident angle in curve 1 is changed from normal incidence to $\theta_{i}$ $=1.2(\mathrm{rad})$. For both cases, we observe significant increases in the heights of the peaks in $R$. The estimated values of $R_{p}$ are also in qualitative agreement with those shown in Fig. 6.

\section{The widths of the enhanced scattering peaks}

Now we discuss the relation between the width of the enhanced scattering peak on the reflection side and the sample thickness $L / l$. For thick samples, where $L / l \gg 1$, it is well known that a CBS cone become narrower when $L / l$ is increased due to presence of longer paths. ${ }^{2,4,6}$ However, this is no longer true in thin samples studied here. In Fig. 7(a) we show the ratio of $I_{r}^{c}\left(0, \theta_{r}\right) / I_{r}^{c}(0,0)$ at various sample thicknesses for the case of normal incidence with $m=1.6$ and 
$k_{0} l=16$. It is interesting to see that the width of CBS cone increases when $L / l$ is increased from 0.02 to 0.1 and then to 0.5 . The trend is reversed to normal behavior found in thick samples when $L / l$ is further increased from 0.5 to 1 and then 3. The inset in Fig. 7(a) plots the full width $w$ at half maximum (FWHM) as a function of $L / l$. A maximum is found when $L / l \cong 0.5$. The anomalous behavior found when $L / l<0.5$ has a different origin. It is mainly due to the relative increase of the probability of lower order scatterings compared to that of higher order scatterings when $L / l$ is increased in thin samples. It has been shown that the width of a CBS cone becomes narrower when higher order scatterings are included. ${ }^{4}$ Thus, an increase in the relative probability of a lower order scattering effectively broadens the CBS cone. To show this, for simplicity, we consider the case of normal incidence and ignore the effects due to internal reflection. The probability of the scattering that experienced by the wave at least once inside the slab is $P_{1}(L) \approx L / l$. For the isotropic scattering, the probability of the scattering that experienced by the wave at least twice inside the slab is $P_{2}(L) \approx(L / l)\left[4 \sin ^{-1}[L /(2 l)] / 2 \pi\right] \approx(L / l)(L / \pi l)$. Similarly, the probability of the scattering that experienced by the wave at least $j$ times inside the slab is $P_{j}(L) \approx(L / l)(L / \pi l)^{j-1}$. When sample thickness $L / l$ is increased by $\delta(L / l)$, the probability $P_{j}(L)$ will increase by $\delta P_{j}(L) \approx j P_{j}(L) /(L / l) \delta(L / l)$. Similarly, we have $\delta P_{j+1}(L) \approx(j+1) P_{j+1}(L) /(L / l) \delta(L / l)$, which can be rewritten as $\delta P_{j+1} \approx[(j+1) / j](l / \pi l) \delta P_{j}(L)$. Thus, when $L / l$ is small we have $\delta P_{j+1}(L)<\delta P_{j}(L)$ for $j$ $\geqslant 1$. This proves our assertion that the probability of lower order scatterings increases more compared to that of higher order scatterings when $L / l$ is increased in thin samples. Furthermore, the presence of the internal reflection effectively increases the sample thickness and an oblique incidence only affects the first order scattering. Thus, our assertion can be extended to include internal reflection and holds for the case of oblique incidence. The width of the enhanced scattering peak on the transmission side has the similar behavior shown in Fig. 7(a) due to the same reason discussed above.

In thick samples, it is also known that the width of a CBS cone is inversely proportional to $k_{0} l$. In Fig. 7(b), we show the the function $I_{r}^{c}\left(0, \theta_{r}\right) / I_{r}^{c}(0,0)$ at different values of $k_{0} l$ for a thin slab with $L / l=0.1$. Similar to the case of thick samples, the width of the CBS cone is found to decrease with increasing $k_{0} l$. However, it does not follow the relation $w$ $\propto\left(k_{0} l\right)^{-1}$ found in thick samples.

The shape of the enhanced scattering peak also depends on the incident angle. In Fig. 8(a), we plot the angular distribution of the intensities $I_{t}^{c}\left(\theta_{i}, \theta_{t}\right)$ and $I_{r}^{c}\left(\theta_{i}, \theta_{r}\right)$ calculated at different incident angles $\theta_{i}$ for the case of $m=3.6, L / l=0.03$, and $k_{0} l=8$. Since the phase factor $\Phi=k L \sqrt{1-\left(\sin \theta_{i} / 3.6\right)^{2}} \leqslant k L=0.864<1$ for all $\theta_{i}^{\prime} s$, the enhanced scattering peaks on the transmission side have almost the same structures as those on the reflection side. The heights of the enhanced scattering peaks decrease like $\cos \theta_{i}$ due to the presence of the factor $\cos \theta_{o}$ in Eq. (17). The FWHM $w$ of the enhanced scattering peak is inversely proportional to $\cos \theta_{i}$ as shown in the inset in Fig. 8(a). This behavior can be obtained from the relation $\mid k \sin \theta_{i}$ $-k \sin \left(\theta_{i}+w / 2\right) \mid \times l \sim 1$, i.e., $w \sim 1 / \cos \theta_{i}$ and holds for thick samples as well.
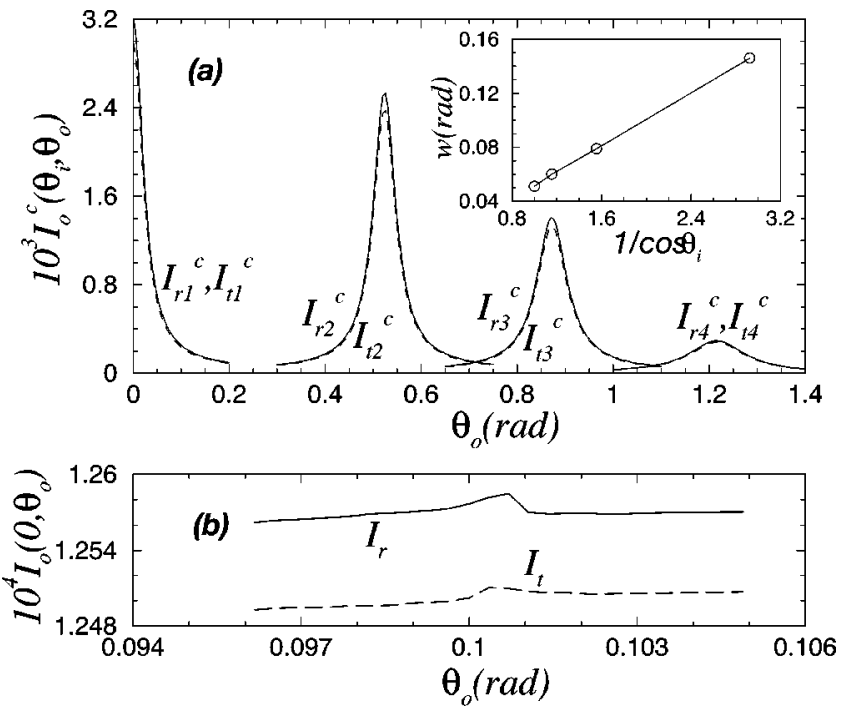

FIG. 8. (a) Intensity $I_{o}^{c}\left(\theta_{i}, \theta_{o}\right)$ of the scattered waves, as the function of the outgoing angle $\theta_{o}$ around the enhanced scattering peaks, with $m=3.6, \quad L / l=0.03, \quad k_{0} l=8, \quad$ and $\theta_{i}=0\left(I_{r 1}^{c}, I_{t 1}^{c}\right)$, $\pi / 6\left(I_{r 2}^{c}, I_{t 2}^{c}\right), 5 \pi / 18\left(I_{r 3}^{c}, I_{t 3}^{c}\right), 7 \pi / 18\left(I_{r 4}^{c}, I_{t 4}^{c}\right)$. The inset gives the relation between the FWHM $w$ and $1 / \cos \theta_{i}$ at given condition in (a). (b) Intensity $I_{o}\left(0, \theta_{o}\right)$ of the scattered waves, as the function of the outgoing angle $\theta_{o}$ around the satellite peaks. $I_{r}=I_{r}\left(0, \theta_{r}\right), I_{t}$ $=I_{t}\left(0, \theta_{t}\right)$, with $m=\sqrt{1.5}, L / l=0.002, k_{0} l=4000$.

\section{Satellite peaks}

Then we discuss the satellite peak of the enhanced scattering. In a thin slab with internal reflection, degenerated eigenmodes with well-confined wavefunctions can exist inside the slab due to total internal reflections. In this case, the $z$ component of the wave vector $\vec{k}$ inside the slab is quantized. A pair of time-reversed paths traveling through different eigenmodes produces a phase difference on both sides of the slab, leading to a satellite peak. The number of degeneracy $M$ is determined by $M \approx m k_{0} /(\pi / L)=k L / \pi=2 L / \lambda$. When $2 L / \lambda>2$, i.e., $L>\lambda$, the Green's function $\left\langle G\left(z_{1}, z_{2} ; q\right)\right\rangle$ in Eq. (7) contains at least two FP resonances and is a peaked function of $q$. The sharpness of the resonance increases with $k_{0} l$. In this case, the enhanced scattering peaks may occur not only near the backscattering and mirror directions of the incident wave $\left(\theta_{r}=\theta_{t}=\theta_{i}\right)$, but also in other directions $\left(\theta_{r}=\theta_{t} \neq \theta_{i}\right)$ both on the reflection and transmission sides. ${ }^{12,13}$

In order to see the satellite peaks within our framework, we choose the same parameters as in Ref. 12, i.e., $m$ $=\sqrt{1.5}, L / l=0.002$ and $k_{0} l=4000$. For normal incidence, the phase factor $\Phi \approx 9.8$ is close to $3 \pi$, at which the main enhanced scattering peak on the transmission side is not very small compared to the main enhanced scattering peak on the reflection side. The heights of the main enhanced scattering peaks on the reflection and transmission sides are, respectively, $\approx 1.4 \times 10^{-5}$ and $\approx 3.9 \times 10^{-6}$. The results of satellite peaks are shown in Fig. 8(b), where the total reflected $\left(I_{r}\right)$ and transmitted $\left(I_{t}\right)$ intensities are plotted as functions of $\theta_{o}$. It can be seen from Fig. 8(b) that satellite peaks occur around $\theta_{r}^{\mathrm{sp}} \approx \theta_{t}^{\mathrm{sp}} \approx 0.10 \mathrm{rad}$. This position is in good agreement with 
the result of Ref. 12. However, the heights of the satellite peaks on the reflection side $\left(\sim 2 \times 10^{-7}\right)$ and transmission side $\left(\sim 4 \times 10^{-8}\right)$ are about two orders of magnitude smaller than their main enhanced scattering peaks. This result is different from that of Ref. 12, where the satellite peaks were found to be about $1 / 3$ of the main peaks. The different is due to the two-dimensional scattering used in Ref. 12 and will be discussed below.

In the above system, we have $k_{0} L / \pi \approx 2.5$ and $k L / \pi$ $\approx 3.1$, implying $M=3$. Thus $\left\langle G\left(z_{1}, z_{2} ; q\right)\right\rangle$ has three peaks. Two of them are eigenmodes at $q_{\alpha} \cong 1.09 k_{0}$ and $q_{\beta} \cong 1.19 k_{0}$. Since both are greater than $k_{0}$, these two modes are well confined in the slab due to total internal reflections. As a result, they produce two very sharp peaks in $\left\langle G\left(z_{1}, z_{2} ; q\right)\right\rangle$. The third one is a quasimode at $q_{\gamma} \cong k_{0}$. It produces a much weaker peak in $\left\langle G\left(z_{1}, z_{2} ; q\right)\right\rangle$. The height of the main enhanced scattering peak corresponds to setting $q=0$ in Eq. (14). In this case, all three peaks of $\left\langle G\left(z_{1}, z_{2} ; q_{1}\right)\right\rangle$ overlap completely with those of $\left\langle G^{*}\left(z_{1}, z_{2} ;\left|\vec{q}-\vec{q}_{1}\right|\right)\right\rangle$ and produce maxima in the functions $A\left(z_{1}, z_{2} ; q\right)$ and $F\left(z_{1}, z_{2} ; q\right)$. The satellite peaks found in Fig. 8(b) arise from the two eigenmodes in the thin slab, i.e, $k_{0}\left|\sin \theta_{o}^{\mathrm{sp}}\right|=\left|q_{\beta}-q_{\alpha}\right| \equiv q^{\mathrm{sp}} \approx 0.10 k_{0}$ and $\theta_{o}^{\mathrm{sp}} \approx \pm 0.10 \mathrm{rad}$. Thus, the height of a satellite peak is determined by the overlap of the peak at $q_{\alpha}$ in $\left\langle G\left(z_{1}, z_{2} ; q_{1}\right)\right\rangle$ with the peak at $q_{\beta}$ in $\left\langle G^{*}\left(z_{1}, z_{2} ;\left|\vec{q}-\vec{q}_{1}\right|\right)\right\rangle$, or vice versa. In an effectively two-dimensional system considered in Ref. 12, the integration of $\vec{q}_{1}$ in Eq. (14) is one dimensional and, therefore, the overlap of peaks at two different eigenmodes can be rather complete. thus, ratio $A\left(z_{1}, z_{2} ; q^{\mathrm{sp}}\right) / A\left(z_{1}, z_{2} ; 0\right)$ is roughly $1 / 3 .{ }^{12}$ However, for the three-dimensional system considered here, the integration of $\vec{q}_{1}$ in Eq. (14) is two dimensional. In this case, the ratio $A\left(z_{1}, z_{2} ; q^{\mathrm{sp}}\right) / A\left(z_{1}, z_{2} ; 0\right)$ is much smaller due to smaller region of overlap between two peaks at $q_{\alpha}$ and $q_{\beta}$ in $\left\langle G\left(z_{1}, z_{2} ; q_{1}\right)\right\rangle$ and $\left\langle G^{*}\left(z_{1}, z_{2} ;\left|\vec{q}-\vec{q}_{1}\right|\right)\right\rangle$, respectively. The satellite peaks may also arise from the degeneracy of one eigenmode and one quasimode. Since the resonant peak of a quasimode is much weaker compared to that of an eigenmode due to its leaky nature, the height of the corresponding peak will be even smaller than the one shown in Fig. 8(b). Such satellite peaks will be difficult to identify numerically.

\section{E. The mean free path in a FP system}

Finally, we should mention that the mean free path, $l$, used in this work is the mean free path of the FP system. Due to the presence of FP interference, the density of states (DOS) of the allowed propagating channels inside the FP system are different from that of the bulk. The properties of impurity scattering are also modified accordingly. Thus, we should expect that $l$ is different from its bulk value $l_{0}$. Below we discuss the effect of the FP interference on $l$. In the 3D bulk limit where $L \rightarrow \infty, l_{0}$ is obtained from the imaginary part of the self-energy $\Sigma$, i.e., $\operatorname{Im~} \Sigma=-k / l_{0}$. However, in the Born approximation with $\delta$-function scattering, ${ }^{10}$ we are able to write $\operatorname{Im} \Sigma=\left(4 \pi / l_{0}\right) \operatorname{Im} G_{3 \mathrm{D}}^{(0)}(\vec{r}, \vec{r} ; k)$, where $G_{3 \mathrm{D}}^{(0)}\left(\vec{r}, \overrightarrow{r^{\prime}} ; k\right)$ $=-\left(1 / 4 \pi\left|\vec{r}-\overrightarrow{r^{\prime}}\right|\right) e^{i k\left|\vec{r}-\vec{r}^{\prime}\right|}$ is the 3D effective medium Green's function and $4 \pi / l_{0}$ represents the two-particle scattering

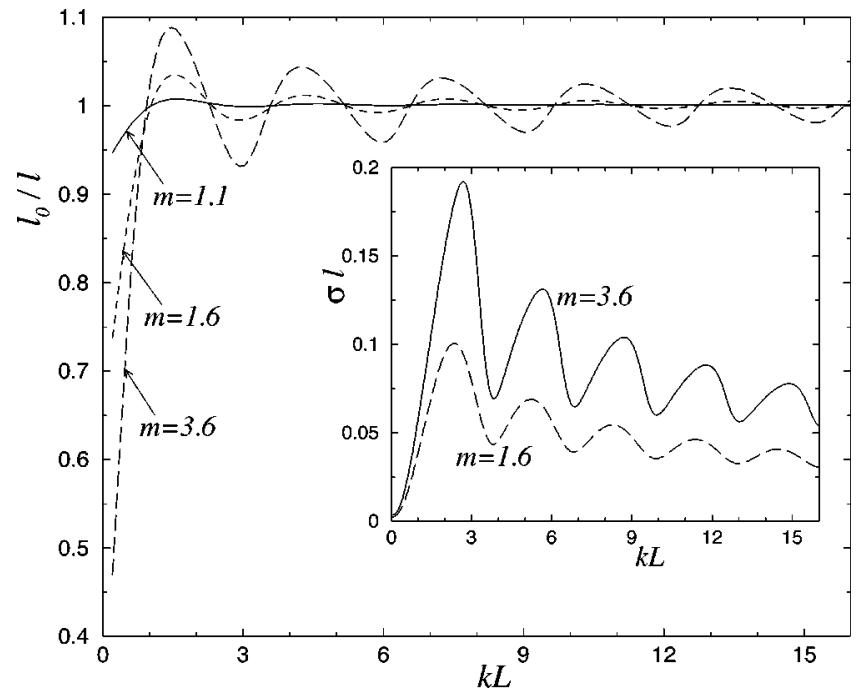

FIG. 9. The ratio $l_{0} / l$, as the function of $k L$, with $m=1.1$ (the solid line), $m=1.6$ (the dashed line), and $m=3.6$ (the long dashed line). The inset gives the relation between $\sigma l$ and $k L$, with $m=1.6$ (the long dashed line) and $m=3.6$ (the solid line)

vertex of the $\delta$-function scattering. The above relation can also be considered as the Ward Identity and is required for the flux conservation in the bulk. ${ }^{2}$ In the case of the FP system, the DOS in the bulk, which is proportional to $\operatorname{Im} G_{3 \mathrm{D}}^{(0)}(\vec{r}, \vec{r} ; k)$, is modified by the FP interference. Therefore $\operatorname{Im} G_{3 \mathrm{D}}^{(0)}(\vec{r}, \vec{r} ; k)$ should be replaced by $\operatorname{Im} G_{\mathrm{FP}}^{(0)}(z, z ; k)$ in the calculation of $l$. Here $G_{\mathrm{FP}}^{(0)}(z, z ; k)$ is the position-dependent Green's function of the FP system and can be written as $G_{\mathrm{FP}}^{(0)}(z, z ; k)=\left(1 / 4 \pi^{2}\right) \int_{0}^{+\infty} G^{(0)}(z, z ; q) 2 \pi q d q$, where $G^{(0)}(z, z ; q)$ has the expression of Eq. (7), but with $i / l$ replaced by $i 0^{+}$. The self-energy term also becomes position dependent, i.e., $\operatorname{Im} \Sigma(z)=\left(4 \pi / l_{0}\right) \operatorname{Im} G_{\mathrm{FP}}^{(0)}(z, z ; k)$. We can define the mean free path $l$ of a FP system through the relation $k / l=-\operatorname{Im}\langle\Sigma(z)\rangle$, where \langle\rangle represents the position average. From Eq. (7), it can be shown that $l_{0} / l$ depends only on $k L$ and $m$. The numerical result of $l_{0} / l$ is shown in Fig. 9 as a function of $k L$ at three different values of $m$. The oscillations shown in $l_{0} / l$ represent the variations in the averaged DOS in the FP system. It is also seen that the amplitude of the oscillations increases with $m$ and dies off gradually when $k L$ becomes large and the system approaches the bulk as expected. Since the self-energy becomes position dependent, we can also define a position-dependent mean free path $l(z)$ through the relation $\operatorname{Im} \Sigma(z)=-k / l(z)$. In Fig. 10, we plot the function $l_{0} / l(z)$ as a function of renormalized position $z / L$ at different values of $k L$ for $m=1.6$ [panel (a)] and $m=3.6$ [panel (b)]. In order to see more clearly the amount of variation in $1 / l(z)$, we calculate the standard deviation $\sigma$ $=\sqrt{\left\langle[1 / l(z)-1 / l]^{2}\right\rangle}$. The results of $\sigma l$ are plotted in the inset of Fig. 9 as functions of $k L$ at $m=1.6$ and 3.6. It is clearly seen that the variation is small when $k L<1$. It reaches a maximum when $k L$ is between 2 and 3 before it decrease to zero when the bulk is approach. For most of the calculations done in this work, the variation of $1 / l(z)$ is within $10 \%$ of its mean value. Thus, our assumption of using a constant value 


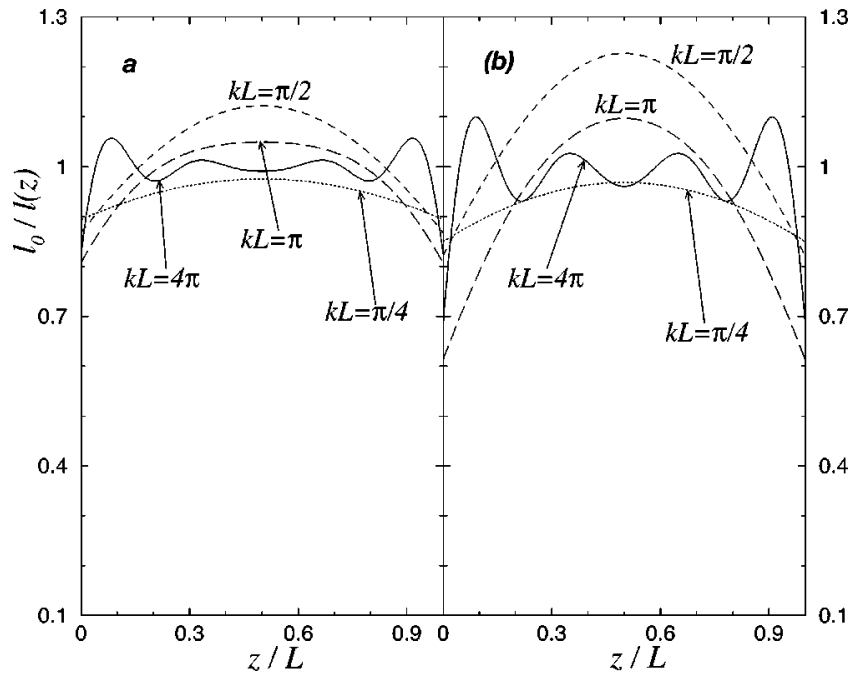

FIG. 10. The quantity $l_{0} / l(z)$, as the function of $z / L . k L=\pi / 4$ (the dotted line), $\pi / 2$ (the dashed line), $\pi$ (the long dashed line), and $4 \pi$ (the solid line), with $m=1.6$ in (a) and $m=3.6$ in (b).

of $1 / l$ in our calculations should be reasonably good. Our results may become less accurate for the case of $m=3.6$ when $k L$ is between 2 and 3 .

\section{CONCLUSION}

In this work, based on the Bethe-Salpeter equation with internal reflection incorporated, we have studied the enhanced scattering peaks on both the reflection and transmission sides of thin disordered slabs after the excitation of a static plane wave incidence. In the Bethe-Salpeter equation, we include the single scattering, the ladder, and the maximally crossed diagrams. We concentrate on the region where $L<l$ and $\lambda<l$. As functions of $L / l$ (or $k_{0} l$ ), the heights of the enhanced scattering peaks on both sides show FP resonances. However, the enhancement factor on the transmission side $E_{t}$ depends mainly on the phase factor $\Phi$ $\equiv k L\left[\sqrt{\left(m^{2}-\sin ^{2} \theta_{i}\right)} / m\right]$. When $\Phi<1$, the enhanced scattering peak on the transmission side has almost the same structure as that on the reflection side and $E_{t}$ is close to 2. When $\Phi>1, E_{t}$ exhibits an oscillatory behavior and approaches one when $\Phi \approx 2.3+n \pi(n=0,1,2, \ldots)$. The amplitudes of the oscillations increase with $m$ and $\theta_{i}$. It is also found that the widths of the enhanced scattering peaks increase with $L$ in thin samples. This is opposite to the well-known trend in thick slabs, where the width of the CBS cone decreases with $L$. This anomalous behavior is due to low order scatterings dominant in thin samples. For the case of $L>\lambda$, at least two eigenmodes with well-confined wave functions can exist inside the slab due to total internal reflections. A pair of multiply scattered wave and its time-reversed counterpart each travels through a different eigenmode can give rise to a satellite peak. We have observed satellite peaks in our calculation. However, due to three-dimensional scatterings, the satellite peaks found here both on the transmission and reflection sides are much weaker in intensity than the main enhanced scattering peaks.

\section{ACKNOWLEDGMENT}

This research is supported by Hong Kong RGC Grant No. HKUST 6058/02P.
${ }^{1}$ A. Ishimaru, Wave Propagation and Scattering in Random Media (Academic, New York, 1987), Vols. I and II.

${ }^{2}$ Scattering and Localization of Classical Waves in Random Media, edited by P. Sheng (World Scientific, Singapore, 1990); P. Sheng, Introduction to Wave Scattering, Localization and Mesoscopic Phenomena (Academic Press, New York, 1995).

${ }^{3}$ M. B. van der Mark, M. P. van Albada, and A. Lagendijk, Phys. Rev. B 37, 3575 (1988).

${ }^{4}$ M. P. Van Albada and A. Lagendijk, Phys. Rev. Lett. 55, 2692 (1985); P. E. Wolf and G. Maret, ibid. 55, 2696 (1985); E. Akkermans, P. E. Wolf, and R. Maynard, ibid. 56, 1471 (1986); M. Kaveh, M. Rosenbluh, I. Edrei, and I. Freund, ibid. 57, 2049 (1986); F. J.P. Schuurmans, M. Megens, D. Vanmaekelbergh, and A. Lagendijk, ibid. 83, 2183 (1999); B. A. van Tiggelen, A. Lagendijk, and D. S. Wiersma, ibid. 84, 4333 (2000); E. Akkermans, P. E. Wolf, R. Maynard, and G. Maret, J. Phys. (France) 49, 77 (1988); R. Berkovits and M. Kaveh, Phys. Rev. B 36, 9322 (1987); I. Freund and R. Berkovits, ibid. 41, 496 (1990); I. Edrei and M. J. Stephen, ibid. 42, 110 (1990); X. Zhang and Z.
Q. Zhang, ibid. 65, 155208 (2002).

${ }^{5}$ A. Lagendijk, R. Vreeker, and P. de Vries, Phys. Lett. A 136, 81 (1989).

${ }^{6}$ J. X. Zhu, D. J. Pine, and D. A. Weitz, Phys. Rev. A 44, 3948 (1991).

${ }^{7}$ P. M. Saulnier and G. H. Watson, Opt. Lett. 17, 946 (1992).

${ }^{8}$ Th. M. Nieuwenhuizen and J. M. Luck, Phys. Rev. E 48, 569 (1993).

${ }^{9}$ A. Yu. Zyuzin, Europhys. Lett. 26, 517 (1994); D. S. Wiersma, M. P. van Albada, and A. Lagendijk, Phys. Rev. Lett. 75, 1739 (1995); S. C. Feng and Z. Q. Zhang, Phys. Rev. B 54, 3240 (1996); W. Deng, D. S. Wiersma, and Z. Q. Zhang, ibid. 56, 178 (1997).

${ }^{10}$ R. Berkovits and S. Feng, Phys. Rev. B 45, 97 (1992).

${ }^{11}$ X. Zhang and Z. Q. Zhang, Phys. Rev. E 66, 016612 (2002).

${ }^{12}$ V. Freilikher, M. Pustilnik, I. Yurkevich, and A. A. Maradudin, Opt. Commun. 110, 263 (1994).

${ }^{13}$ V. Freilikher, M. Pustilnik, and I. Yurkevich, Phys. Lett. A 193, 467 (1994). 